\title{
ON EXTENDING NONVANISHING SEMICHARACTERS
}

\author{
W. W. COMFORT ${ }^{1}$ AND PAUL HILL
}

Introduction. Let $\chi$ be a semicharacter whose domain is a subsemigroup $S$ of a commutative semigroup $T$, and suppose that the pair $(\chi, S)$ satisfies a property $(P)$. One may ask for conditions on $(\chi, S)$ and/or $T$ necessary and/or sufficient that there exist a semicharacter $\psi$ with domain $T$, agreeing with $\chi$ on $S$ and for which $(\psi, T)$ satisfies $(P)$. Of the results in the literature of this genre, the most satisfactory are those of Kenneth A. Ross in [1] and [2]. We use Ross' results (quoted explicitly below) to deal in the present paper with the property $(P)$ for which (by definition) $(\chi, S)$ has $(P)$ if and only if $\chi$ vanishes nowhere on $S$.

Notation, definitions, and Ross' results. A semicharacter on a commutative semigroup $T$ is a homomorphism from $T$ into the multiplicative complex unit disk, not vanishing identically. A semicharacter $\psi$ on $T$ is called a character if $|\psi(x)|=1$ for each $x$ in $T$. If $\chi$ is a semicharacter on a subsemigroup $S$ of $T$, and if $\psi$ agrees with $\chi$ on $S$, then we say that $(\psi, T)$ is a semicharacter extension of $(\chi, S)$. Our aim, as suggested above, is to construct $\psi$, given $T$ and $S$ and $\chi$.

Following Ross in [1] and [2], we use the symbols (*), (A) and (iii) as follows:

$\left(^{*}\right) \quad(a, b, x) \in S \times S \times T$ and $a x=b x$ imply $\chi(a)=\chi(b)$;

(A) $(a, b, x) \in S \times S \times T$ and $a x=b$ imply $|\chi(a)| \geqq|\chi(b)|$;

(iii) $(a, b, x, y) \in S \times S \times T \times T$ and $a x y=b y$ imply $|\chi(a)| \geqq|\chi(b)|$.

THEOREM (FROM [1]). In order that $(\chi, S)$ admit a semicharacter extension $(\psi, T)$, it is necessary and sufficient that (A) hold.

TheOREM (FROM [2]). In order that $(\chi, S)$ admit an extension $(\psi, T)$ with $\psi$ a character on $T$, it is necessary and sufficient that ( $\chi$ be a character on $S$ and that) $\left(^{*}\right)$ hold.

Example (From [2]). There is a pair $(\chi, S)$ satisfying $\left({ }^{*}\right)$ and (iii) and with $\chi$ vanishing nowhere on $S$, with the property that $\psi$ vanishes somewhere on $T$ whenever $(\psi, T)$ is a semicharacter extension of $(\chi, S)$.

Received by the editors December 9, 1964 and, in revised form, June 2, 1965.

1 This author acknowledges support of the National Science Foundation under contracts NSF-GP 2200 and NSF-GP 5750. 
Technical lemmas. Suppose now that $\chi$ vanishes nowhere on $S$. We will show in what follows that in order that $(\chi, S)$ admit a semicharacter extension $(\psi, T)$, and $\psi$ vanishing nowhere on $T$, it suffices that $\left(^{*}\right)$ hold and that for each $x$ in $T$ we have

$$
S T x z \cap S z \neq \varnothing \quad \text { for some } z \text { in } T \text {. }
$$

The first condition, $\left({ }^{*}\right)$, depends upon both the values assumed by $\chi$ and the multiplicative structure of $T$; the second condition depends only upon how $S$ is positioned inside $T$. In all that follows, we shall assume that the pair $(\chi, S)$ satisfies both these conditions, as well as condition (iii) and the condition that $\chi$ vanishes nowhere on $S$. None of these conditions is destroyed or created by the adjunction to $T$ of an identity element, so we shall henceforth assume that there is in $T$ an identity element $e$, and in fact that $e \in S$.

We begin with a lemma, possibly unexpected but not truly essential for what follows, which reduces the problem under consideration to the corresponding one for positive semicharacters.

LEMMA 1. In order that $(\chi, S)$ admit a semicharacter extension $(\psi, T)$ with $\psi$ vanishing nowhere on $T$, it is necessary and sufficient that $(|\chi|, S)$ admit a semicharacter extension $(\omega, T)$ with $\omega$ vanishing nowhere on $T$.

Proof. Necessity is obvious. For sufficiency, note that $\left(^{*}\right)$ holds for $\chi /|\chi|$ on $T$, so that some character $\chi^{\prime}$ extends $\chi /|\chi|$ to $T$. The desired extension $(\psi, T)$ of $(\chi, S)$ is given by the relation $\psi=\chi^{\prime} \omega$ or by $\psi=\chi^{\prime}|\omega|$.

In what follows, the symbols $k, l, m, n$ denote positive integers. The symbol $r^{1 / n}$ will appear only when $r$ is a real, positive number, and it represents that unique positive real number $s$ for which $s^{n}=r$.

Definition. For $x$ in $T$ let

$$
\begin{aligned}
\alpha(x)=\sup \left\{|\chi(a) / \chi(b)|^{1 / n}:\right. & \\
b x^{n} y z & =a z \text { with }(a, b, y, z) \in S \times S \times T \times T\} .
\end{aligned}
$$

REMARK. For each $x$ in $T$ we have by hypothesis $b x y z=a z$ for some $(a, b, y, z) \in S \times S \times T \times T$. Hence $\alpha(x)>0$. That always $\alpha(x) \leqq 1$ is a restatement of condition (iii).

Definition. For each nonvanishing semicharacter extension $(\psi, R)$ of $(\chi, S)$ let $f_{\psi}$ be defined on $T$ by the rule

$$
\begin{aligned}
& f_{\psi}(x)=\inf \left\{|\psi(a) /(\psi(b) \alpha(y))|^{1 / n}:\right. \\
& \left.\quad b x^{n} y z=a w z \text { with }(a, b, w, y, z) \in R \times R \times T \times T \times T\right\} .
\end{aligned}
$$


We now develop the technical properties of the functions $\alpha$ and $f_{\downarrow}$ which will yield the proof of our theorem.

Lemma 2. The function $\alpha$ enjoys the following properties:

(a) if $\left(x_{1}, x_{2}\right) \in T \times T$, then $\alpha\left(x_{1}\right) \geqq \alpha\left(x_{1} x_{2}\right) \geqq \alpha\left(x_{1}\right) \alpha\left(x_{2}\right)$;

(b) if $\left(x_{1}, x_{2}, x_{3}\right) \in T \times T \times T$ and $x_{1} x_{3}=x_{2} x_{3}$, then $\alpha\left(x_{1}\right)=\alpha\left(x_{2}\right)$;

(c) $\alpha$ agrees with $|\chi|$ on $S$ (in particular, $\alpha(e)=1$ ).

Proof. (a) The first inequality is obvious. If for $1 \leqq k \leqq 2$ and for positive integers $m$ and $n$ we have

$$
b_{1} x_{1}^{m} y_{1} z_{1}=a_{1} z_{1} \text { and } b_{2} x_{2}^{n} y_{2} z_{2}=a_{2} z_{2},
$$

then it follows that

$$
b_{1}^{n} b_{2}^{m}\left(x_{1} x_{2}\right)^{m n} y_{1}^{n} y_{2}^{m} z_{1} z_{2}^{m}=a_{1}^{n} a_{2}^{m} z_{1}^{n} z_{2}
$$

whence we have

$$
\alpha\left(x_{1} x_{2}\right) \geqq\left|\chi\left(a_{1}\right) / \chi\left(b_{1}\right)\right|^{1 / m}\left|\chi\left(a_{2}\right) / \chi\left(b_{2}\right)\right|^{1 / n}
$$

and then the second inequality in (a).

(b) The relation $\alpha\left(x_{1}\right) \leqq \alpha\left(x_{2}\right)$ follows from the observation that if $b x^{n} y z=a z$ then

$$
b x_{2}^{n} y\left(x_{3}^{n} z\right)=a\left(x_{3}^{n} z\right),
$$

so that $\alpha\left(x_{2}\right) \geqq|\chi(a) / \chi(b)|^{1 / n}$. A similar argument gives $\alpha\left(x_{2}\right) \leqq \alpha\left(x_{1}\right)$.

(c) The inequality $|\chi| \geqq \alpha$ on $S$ follows from condition (iii). The identity $x x e e=x^{2} e$ yields the relation

$$
\alpha(x) \geqq\left|\chi\left(x^{2}\right) / \chi(x)\right|=|\chi(x)|
$$

for each $x$ in $S$.

Lemma 3. If $(\psi, R)$ is a nonvanishing semicharacter extension of $(\chi, S)$ for which $f_{\psi} \geqq \alpha$ throughout $T$, then $f_{\psi}$ enjoys the following properties:

(a) if $\left(x_{1}, x_{2}\right) \in T \times T$, then $f_{\psi}\left(x_{1} x_{2}\right) \leqq f_{\psi}\left(x_{1}\right)$;

(b) $f_{\psi}\left(x^{k}\right)=\left(f_{\psi}(x)\right)^{k}$ for each $x$ in $T$ and each integer $k>0$;

(c) if $(x, c) \in T \times R$, then $f_{\psi}(x c)=f_{\psi}(x)|\psi(c)|$;

(d) if $\left(x_{1}, x_{2}, x_{3}\right) \in T \times T \times T$ and $x_{1} x_{3}=x_{2} x_{3}$, then $f_{\psi}\left(x_{1}\right)=f_{\psi}\left(x_{2}\right)$;

(e) $f_{\psi}$ agrees with $|\psi|$ on $R$.

Proof. (a) This is obvious, since if $b x^{n} y z=a w z$, then $b\left(x_{1} x_{2}\right)^{n} y z$ $=a\left(w x_{2}^{n}\right) z$.

(b) If $b\left(x^{k}\right)^{n} y z=a w z$, then $f_{\psi}(x) \leqq|\psi(a) /(\psi(b) \alpha(y))|^{1 / k n}$, whence $\left(f_{\psi}(x)\right)^{k} \leqq f_{\psi}\left(x^{k}\right)$. 
If $b x^{n} y z=a w z$, then $b^{k}\left(x^{k}\right)^{n} y^{k} z^{k}=a^{k} w^{k} z^{k}$, so that

$$
\begin{aligned}
f_{\psi}\left(x^{k}\right) & \leqq\left|\psi\left(a^{k}\right) /\left(\psi\left(b^{k}\right) \alpha\left(y^{k}\right)\right)\right|^{1 / n} \leqq|\psi(a) /(\psi(b) \alpha(y))|^{k / n} \\
& =\left[|\psi(a) /(\psi(b) \alpha(y))|^{1 / n}\right]^{k},
\end{aligned}
$$

whence $f_{\psi}\left(x^{k}\right) \leqq\left(f_{\psi}(x)\right)^{k}$.

(c) If $b(x c)^{n} y z=a w z$, then $\left(b c^{n}\right) x^{n} y z=a w z$, so that

$$
\begin{aligned}
f_{\psi}(x) & \leqq\left|\psi(a) /\left(\psi\left(b c^{n}\right) \alpha(y)\right)\right|^{1 / n} \\
& =|\psi(a) /(\psi(b) \alpha(y))|^{1 / n}(1 /|\psi(c)|),
\end{aligned}
$$

whence $f_{\psi}(x)|\psi(c)| \leqq f_{\psi}(x c)$.

If $b x^{n} y z=a w z$, then $b(x c)^{n} y z=a c^{n} w z$, so that

$$
\begin{aligned}
f_{\psi}(x c) & \leqq\left|\psi\left(a c^{n}\right) /(\psi(b) \alpha(y))\right|^{1 / n} \\
& =|\psi(a) /(\psi(b) \alpha(y))|^{1 / n}|\psi(c)|,
\end{aligned}
$$

whence $f_{\psi}(x c) \leqq f_{\psi}(x)|\psi(c)|$.

(d) This easy proof follows a pattern very similar to that given in (b) of Lemma 2.

(e) From (b) and the condition $f_{\psi}(e) \geqq \alpha(e)=1$ it follows that $f_{\psi}(e)=1$, so (c) yields (e).

\section{The extension theorem.}

THEOREM. Let the semicharacter $\chi$ vanish nowhere on $S$, let conditions $\left.{ }^{*}\right)$ and (iii) be satisfied, and suppose that for each $x$ in $T$ there exists $(a, b, y, z) \in S \times S \times T \times T$ such that bxyz=az. Then $(\chi, S)$ admits $a$ semicharacter extension $(\psi, T)$, with $\psi$ vanishing nowhere on $T$.

Proof. In view of Lemma 1 we may suppose that $\chi$ is positive on $S$. Let

$$
\begin{array}{r}
z=\{(\psi, R):(\psi, R) \text { is a positive semicharacter extension of }(\chi, S) \\
\text { and } \left.f_{\psi} \geqq \alpha\right\} .
\end{array}
$$

We define on $z$ a partial ordering as follows:

$$
\left(\psi_{1}, R_{1}\right) \leqq\left(\psi_{2}, R_{2}\right) \text { if }\left(\psi_{2}, R_{2}\right) \text { extends }\left(\psi_{1}, R_{1}\right) .
$$

Since each chain in $z$ obviously admits an upper bound in $z$ we can complete the proof by showing first that $(\chi, S) \in_{z}$ and second that any maximal element $(\psi, R)$ of $z$ satisfies the relation $R=T$.

If $b x^{n} y z=a w z$ with $(a, b, w, y, z) \in S \times S \times T \times T \times T$, then

$$
\begin{aligned}
\chi(b)(\alpha(x))^{n} \alpha(y) & =\alpha(b)(\alpha(x))^{n} \alpha(y) \leqq \alpha\left(b x^{n} y\right) \\
& =\alpha(a w) \leqq \alpha(a)=\chi(a),
\end{aligned}
$$


so that $\alpha(x) \leqq|\chi(a) /(\chi(b) \alpha(y))|^{1 / n}$. The relation $\alpha \leqq f_{\chi}$ follows; hence $(\chi, S) \in$.

Suppose now that $(\psi, R)$ is maximal in $z$ and that there exists $x_{0} \in T \backslash R$. Denoting by $R^{*}$ the semigroup generated in $T$ by $R$ and $x_{0}$, and by $\psi^{*}$ the restriction to $R^{*}$ of $f_{\psi}$, we will show

(a) $\psi^{*}$ is multiplicative on $R^{*}$;

(b) $\psi^{*}$ is a semicharacter on $R^{*}$;

(c) $\psi^{*}$ vanishes nowhere on $R^{*}$; and

(d) $f_{\psi^{*}} \geqq \alpha$.

Lemma 3 handles the trivial cases of (a). For the nontrivial case, let $c_{1} \in R$ and $c_{2} \in R$. Then

$$
\begin{aligned}
\psi^{*}\left(\left(x_{0}^{k} c_{1}\right)\left(x_{0}^{l} c_{2}\right)\right) & =f_{\psi}\left(\left({ }_{0}^{k} c_{1}\right)\left(x_{0}^{l} c_{2}\right)\right) \\
& =f_{\psi}\left(x_{0}^{k+l} c_{1} c_{2}\right)=f_{\psi}\left(x_{0}^{k+l}\right) \psi\left(c_{1}\right) \psi\left(c_{2}\right) \\
& =\left(f_{\psi}\left(x_{0}^{k}\right) \psi\left(c_{1}\right)\right)\left(f_{\psi}\left(x_{0}^{l}\right) \psi\left(c_{2}\right)\right) \\
& =\psi^{*}\left(x_{0}^{k} c_{1}\right) \psi^{*}\left(x_{0}^{l} c_{2}\right) .
\end{aligned}
$$

For (b), we note that $\psi^{*}$ agrees with $\psi$ on $R$, so that $\psi^{*}$ does not vanish identically on $R^{*}$. To see that $\psi^{*}$ maps $R^{*}$ into the unit disk we fix $x$ in $T$ and observe that the relation $b x y z=a w z$ holds upon choosing $a=b=y=z=e \in R$ and $w=x$. Thus if $x \in R^{*}$ we have

$$
\left|\psi^{*}(x)\right|=\psi^{*}(x)=f_{\psi}(x) \leqq|\psi(e) /(\psi(e) \alpha(e))|=1 .
$$

For (c) we have only to recall that $\psi^{*}=f_{\psi} \geqq \alpha>0$ throughout $R^{*}$.

For (d), suppose that $\left(x_{0}^{k} b\right) x^{n} y z=\left(x_{0}^{l} a\right) w z$ with $(a, b, w, y, z) \in R \times R$ $\times T \times T \times T$.

If $k<l$, then

$$
b x^{n} y(\stackrel{k}{k} z)=a\left(x_{0}^{l-k} w\right)\left(\stackrel{k}{x_{0} z}\right)
$$

so that

$$
\begin{aligned}
\psi(b)(\alpha(x))^{n} \alpha(y) & \leqq \psi(b) \alpha\left(x^{n} y\right) \leqq \psi(b) f_{\psi}\left(x^{n} y\right) \\
& =f_{\psi}\left(b x^{n} y\right)=f_{\psi}\left(a x_{0}^{l-k} w\right) \\
& \leqq f_{\psi}\left(a x_{0}^{l-k}\right)=\psi^{*}\left(a x_{0}^{l-k}\right)
\end{aligned}
$$

whence

$$
\alpha(x) \leqq\left|\psi^{*}\left(a x_{0}^{l-k}\right) /(\psi(b) \alpha(y))\right|^{1 / n}=\left|\psi^{*}\left(x_{0}^{l} a\right) /\left(\psi^{*}\left(x_{0}^{k} b\right) \alpha(y)\right)\right|^{1 / n} .
$$

If $k=l$, then

$$
b x^{n} y\left(x_{0}^{k} z\right)=a w\left(x_{0}^{k} z\right)
$$


so that

$$
\alpha(x) \leqq|\psi(a) /(\psi(b) \alpha(y))|^{1 / n}=\left|\psi^{*}\left(x_{0}^{l} a\right) /\left(\psi^{*}\left(x_{0}^{k} b\right) \alpha(y)\right)\right|^{1 / n} .
$$

If $k>l$, then

$$
b x_{0}^{k-l}\left(x^{n} y\right)\left(x_{0}^{l} z\right)=a w\left(x_{0}^{l} z\right)
$$

so that

$$
\begin{aligned}
\left(\psi^{*}\left(x_{0}\right)\right)^{k-l} & =\left(f_{\psi}\left(x_{0}\right)\right)^{k-l} \leqq\left|\psi(a) /\left(\psi(b) \alpha\left(x^{n} y\right)\right)\right| \\
& \leqq\left|\psi(a) /\left(\psi(b)(\alpha(x))^{n} \alpha(y)\right)\right|
\end{aligned}
$$

whence

$$
\begin{aligned}
\alpha(x) & \leqq \mid \psi^{*}(a) /\left(\left.\psi^{*}\left(x_{0}^{k-l} b\right) \alpha(y)\right|^{1 / n}\right. \\
& =\left|\psi^{*}\left(x_{0}^{l} a\right) /\left(\psi^{*}\left(x_{0}^{k} b\right) \alpha(y)\right)\right|^{1 / n} .
\end{aligned}
$$

The inequality $f_{\psi^{*}} \geqq \alpha$ now follows, so the proof is complete.

REMARKs. We acknowledge with gratitude a penetrating comment of the referee which led to the statement of our theorem in the form given above, slightly more pleasing than the original. The referee's (apparent) suggestion that the proof can be drastically simplified by omitting reference to the functions $f_{\psi}$ and proving simply that the function $\alpha$ is itself an extension to $T$ of the desired form for $(\chi, S)$ is, however, not to be countenanced. The following example shows that the function $\alpha$ need not be multiplicative on $T$.

EXAmple. Let $T$ be the free Abelian semigroup on the two symbols $a$ and $b$, and let $S$ denote the subsemigroup of $T$ consisting of all positive integral powers of $a b$. If $\chi$ is defined on $S$ by the relation

$$
\chi\left((a b)^{n}\right)=1 / 2^{n},
$$

then all hypotheses of our theorem are satisfied (once an identity is adjoined to $T$ ), but $\alpha(a)=\alpha(b)=\alpha(a b)=1 / 2$.

\section{REFERENCES}

1. K. A. Ross, $A$ note on extending semicharacters on semigroups, Proc. Amer. Math Soc. 10 (1959), 579-583.

2. —_ Extending characters on semigroups, Proc. Amer. Math. Soc. 12 (1961), 988-990.

UNIVERSITY OF ROCHESTER AND

EMORY UNIVERSITY 